\title{
A spectrophotometric study of RW Trianguli
}

\author{
P. J. Groot ${ }^{1,2}$, R. G. M. Rutten ${ }^{3}$, and J. van Paradijs ${ }^{2,4}$ \\ 1 Department of Astrophysics, University of Nijmegen, PO Box 9010, 6500 GL, Nijmegen, The Netherlands \\ 2 Astronomical Institute "Anton Pannekoek"/ CHEAF, Kruislaan 403, 1098 SJ, Amsterdam, The Netherlands \\ 3 Isaac Newton Group of Telescopes, Apartado de Correos 321, 38700, Sta Cruz de La Palma, Islas Canarias, Spain \\ ${ }^{4}$ Physics Department, UAH, Huntsville, AL 35899, USA
}

Received 10 October 1999 / Accepted 23 December 2003

\begin{abstract}
On the basis of spectrophotometric observations we reconstruct the accretion disk of the eclipsing novalike cataclysmic variable RW Tri in the wavelength region 3600-7000 $\AA$. We find a radial temperature profile that is, on average, consistent with that expected on the basis of the theory of optically thick, steady state accretion disks and infer a mass-accretion rate in RW Tri of $\sim 10^{-8} M_{\odot} \mathrm{yr}^{-1}$. The line emission is dominated by two areas: one around the hot-spot region and one near the white dwarf. Both emission regions have appreciable vertical extension, and seem to be decoupled from the velocity field in the disk. In our observations RW Tri shows a number of features that are characteristic of the SW Sex sub-class of novalike stars. The appearance of a novalike system as a UX UMa/RW Tri or SW Sex star seems to be mainly governed by the mass-transfer rate from the secondary at the time of observation.
\end{abstract}

Key words. accretion, accretion disks - stars: binaries: eclipsing - stars: individual: RW Tri stars: novae, cataclysmic variables

\section{Introduction}

RW Tri is one of the earliest known cataclysmic variables (CVs), discovered by Protitch (1937). It has been studied extensively photometrically, but has been largely neglected in spectroscopic studies. To our knowledge only two extensive optical spectrophotometric studies, by Kaitchuck et al. (1983) and Still et al. (1995) have been made of this system. RW Tri is generally assumed to be a standard novalike CV (see Warner 1995 for a general overview of CVs), but both spectroscopic studies have shown that the phase depedence and the light curves of the emission lines show features that are difficult to explain in a standard CV picture.

RW Tri was included in the broad-band photometry eclipse mapping studies of Rutten et al. (1992) who showed that the radial temperature profile of its accretion disk is consistent with the $T \propto R^{-3 / 4}$ dependence expected on the basis of accretion disk theory (see e.g. Frank et al. 1992). RW Tri is in this respect similar to UX UMa that has been shown spectrophotometrically to follow the same temperature profile (Rutten et al. 1993, 1994).

RW Tri is also known to undergo irregular brightness variations of up to one magnitude in its out-of-eclipse brightness, as was first shown by Walker (1963). It has even been observed (Still et al. 1995) to be more than three magnitudes brighter than its "quiescent" value at $A B \sim 13.5$. This irregular

Send offprint requests to: P. J. Groot, e-mail: pgroot@astro.kun.nl behaviour is not unique for RW Tri (see e.g. the recent results on GS Pav; Groot et al. 1998), although it is the best documented case.

\section{Observations}

On the nights of 22-26 October 1994, we obtained a total of 671 low-resolution ( $8 \AA$ ) spectra using the Intermediate Dispersion Spectrograph with the R300V grating and a $1 \mathrm{k} \times 1 \mathrm{k}$ TEK CCD, attached to the $2.5 \mathrm{~m}$ Isaac Newton Telescope on the island of La Palma. A wide slit (2'.5) and a second star on the slit (48" NW of RW Tri) were used to obtain differential photometry. For absolute flux calibration the spectral flux standard BD +28 4211 (Oke 1990) was used with a 5" wide slit for the standard as well as for RW Tri and its comparison star.

All spectra were obtained with a $50 \mathrm{~s}$ on-target integration time. With a $\sim 60 \mathrm{~s}$ dead-time for CCD readout and data storage, we obtained an effective time resolution of $110 \mathrm{~s}$, or $1 / 182 \mathrm{nd}$ of the orbital period. A total of five eclipses were observed. Throughout the nights $\mathrm{CuAr}$ arc spectra were taken for wavelength calibration. All data were reduced in the standard fashion using the ESO-MIDAS package, with additionally written software and they were optimally extracted (Horne 1986).

Based on the colour excess given by Rutten et al. (1992) of $E(B-V)=0.1$ we have dereddened all our spectra using the galactic reddening coefficients given by Cardelli et al. (1990), assuming a standard $R_{V}=3.1$. 
Table 1. System parameters of RW Tri.

\begin{tabular}{ll}
\hline \hline Period & $20034.717 \mathrm{~s}$ \\
$M_{\mathrm{WD}}$ & $0.7 M_{\odot}$ \\
$M_{\mathrm{sec}}$ & $0.6 M_{\odot}$ \\
Inclination & $75^{\circ}$ \\
Distance & $330 \mathrm{pc}$ \\
\hline
\end{tabular}

\section{Ephemeris and system parameters}

We have phase folded all spectra using the ephemeris given by Robinson et al. (1991). Trial eclipse maps using the system parameters given in Table 1 showed a shift in the phase of minimum light. Shifting the phases by -0.0046 of an orbital period, as has been found before by Smak (1995), corrected for this. A revised ephemeris is given in Eq. (1)

$$
\mathrm{HJD}_{\text {mid_ecl }}=2441129.36380(10)+0.231883297 E \text {. }
$$

The system parameters of RW Tri are rather uncertain, especially the $(q, i)$ pair, where $q$ is the mass ratio $\left(M_{2} / M_{1}\right)$ and $i$ the orbital inclination. Values for $i$ range between $67^{\circ}$ (Kaitchuck et al. 1983) to as high as $80^{\circ}$ (Mason et al. 1997), with the component masses varying accordingly. Previous studies agree that the mass ratio is close to unity. We have chosen to use the values as given in Rutten et al. (1992, see also Table 1).

\section{Continuum light curves}

The continuum light curves of RW Tri (Fig. 1) show that the system varied up to $\sim 30 \%$ in its out-of-eclipse light level from night to night. These short term variations of RW Tri have been long known (Walker 1963; Smak 1995). In our observations the system varied, at $4500 \AA$, between $A B=13.2(18.5 \mathrm{mJy})$ on the night of October 23, to $A B=12.9$ (23 mJy) on October 26 .

The light curves show the eclipse as the most prominent feature. As in other novalike systems, only a weak orbital hump is seen before the eclipse. The eclipse shape itself, however, does show a prominent hot-spot egress feature.

\section{Spectral line behaviour}

\subsection{Average spectrum}

In Fig. 2 we show the average flux calibrated spectrum of RW Tri during our observations. The spectrum shows the usual emission lines of H I, He I and He II. All lines appear single peaked in our low-resolution spectra. In the average, non hotspot, non mid-eclipse, spectrum (middle curve in Fig. 2) we can see absorption troughs underlying the Balmer series, especially well visible in the higher members, $\mathrm{H} \gamma$ and higher. These troughs become deeper when the hot-spot is visible (top spectrum in Fig. 2). The red part of the spectrum shows the Earth's atmospheric features at $6300 \AA$ and $6900 \AA$.

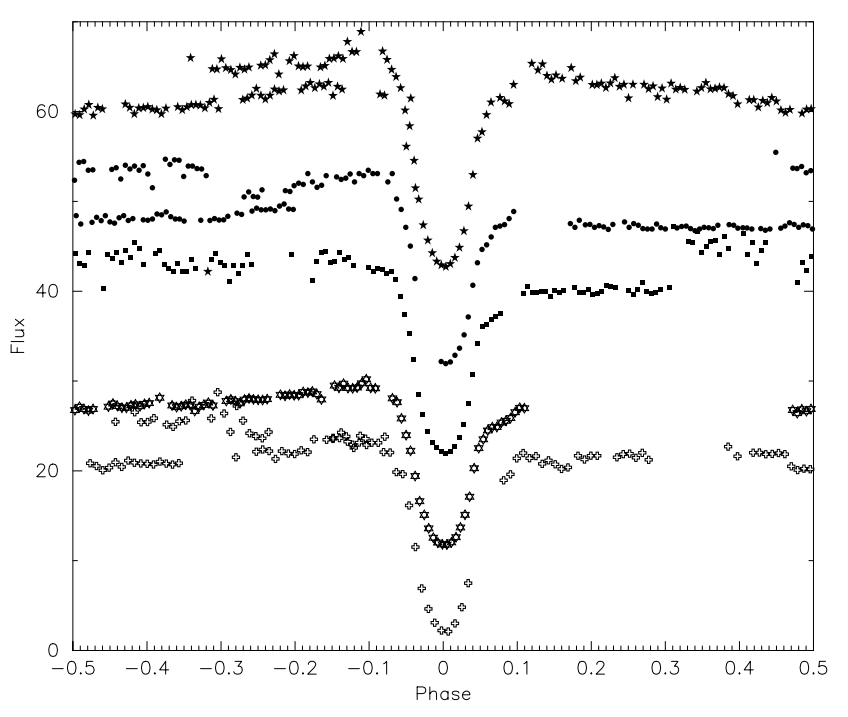

Fig. 1. The continuum light curves of RW Tri between $4400 \AA$ and $4600 \AA$, from bottom (22 October) to top (26 October). The light curves are offset by steps of $10 \mathrm{mJy}$ per night, with no offset for the bottom curve.

\subsection{Trailed spectra}

In Fig. 3 we show the trailed spectra of the main lines in RW Tri after having subtracted the continuum contribution by making a linear fit to the adjacent continuum.

In Fig. 3 we see that most of the lines consist of at least two components, best seen in the He I $\lambda 6678$ line. A component with very low radial velocity amplitude dominates the lower Balmer $(\mathrm{H} \alpha, \mathrm{H} \beta)$, the He II $\lambda 4686$ and lower He I lines. In all these lines a second component, with higher radial velocity is also seen. It is this second component that is the only one seen, and in absorption, in the He I $\lambda 4026$ and $\lambda 4471$ lines. The lower He I lines show this component in emission. We will use the $\mathrm{H} \alpha$ and $\mathrm{He}$ II $\lambda 4686$ lines to derive the phasing and amplitude of the low-velocity component since this component dominates in these lines. The He I $\lambda 4471$ absorption line will be used to derive the radial velocity and phasing of the higher velocity component. We will here make the assumption that the amplitude and phase of these two components is the same in all the lines.

\subsubsection{Radial velocity curves}

We have used an auto-correlation technique to determine the radial velocity curve of the low-velocity component dominating $\mathrm{H} \alpha$ and $\mathrm{He}$ II $\lambda$ 44686. In Fig. 5 we show the radial velocity curve and a sinusoidal fit to the phase interval $0.1<\varphi<0.9$. Both $\mathrm{H} \alpha$ and He II $\lambda 4686$ show the same phase lag and radial velocity amplitude, indicating that they are formed in the same region. The phase lag shows that the emission site is not on the line of centers, connecting the secondary, the center-of-mass of the system and the white dwarf.

The radial velocity curve of He I $\lambda 4471$ (Fig. 6) shows that the high-velocity component is in anti-phase with the secondary star and has an amplitude of $\sim 330 \mathrm{~km} \mathrm{~s}^{-1}$. 


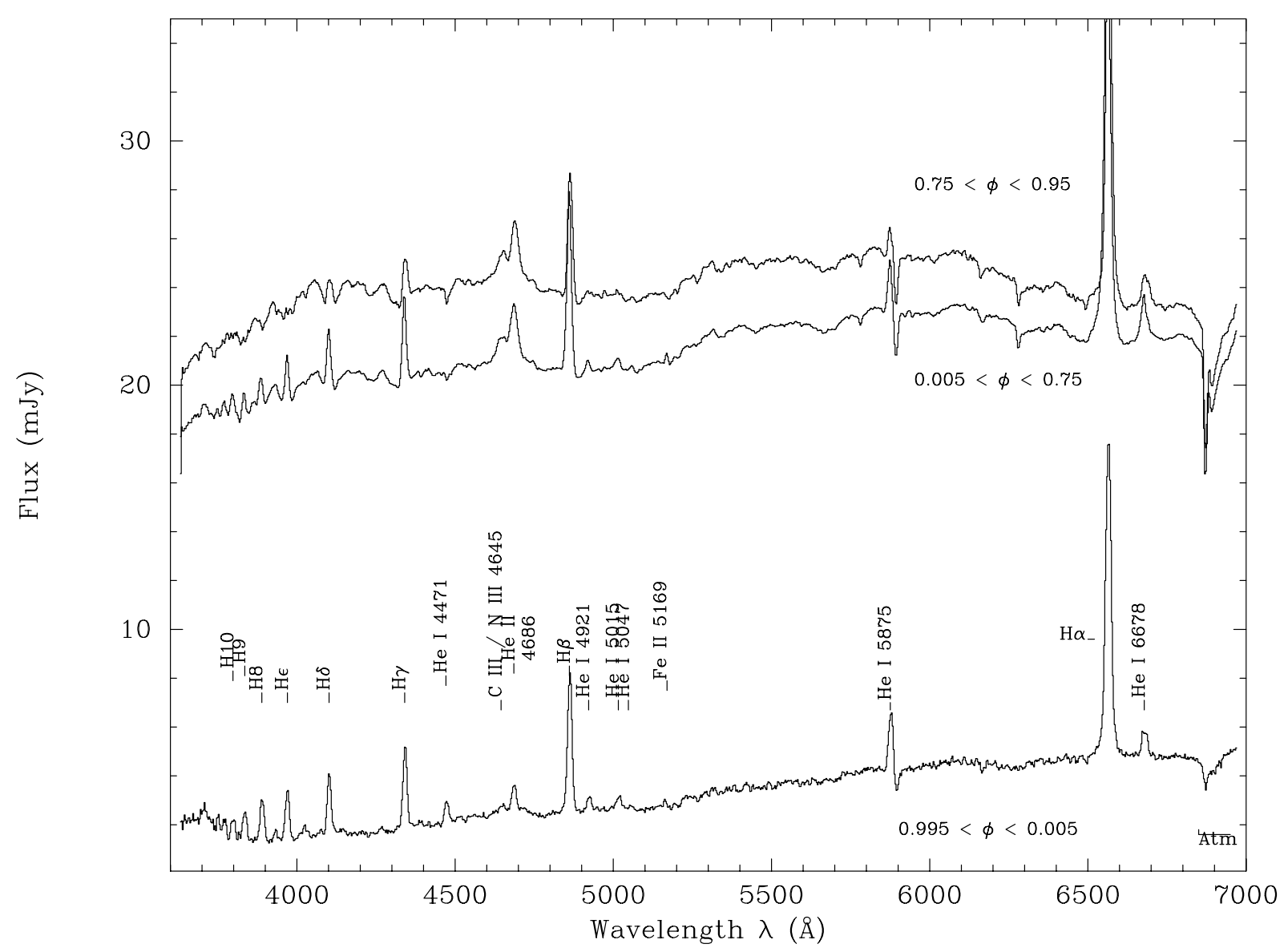

Fig. 2. The average spectrum of RW Tri, divided in three phase intervals. The bottom curve shows the spectrum in mid-eclipse $(0.995<\varphi<$ 0.005), the middle curve the spectrum outside eclipse and outside the phase interval that a hot-spot can be visible $(0.005<\varphi<0.75)$ and the top curve shows the spectrum during the hot-spot phase $(0.75<\varphi<0.95)$.

Smith et al. (1993) have shown the secondary to be a K7-type star. Among the most prominent lines in late K-type stars is the Ca I triplet at $6200 \AA$.

In Fig. 7 we show a trailed spectrum of the region around $6160 \AA$. We see that indeed there are absorption lines present, at $\sim 6160 \AA$ at $\sim 6120 \AA$ and a trace can be seen of a third line around $6100 \AA$. These three wavelengths uniquely identify this set of lines as the CaI triplet of $\lambda 6102, \lambda 6122$ and $\lambda 6162$.

We have determined the radial velocity curve of the secondary by auto-correlating the absorption profile of the $\lambda 6162$ line at all phases with the profile between phase $0.65<$ $\varphi<0.7$ (Fig. 4). A sinusoidal fit to the phase interval $0.1<\varphi<$ 0.9 shows that the phasing of the line coincides with that of the secondary star and the derived amplitude is consistent with that derived by Smith et al. (1993) from the near infrared Na I lines. The strength of the absorption lines reaches a minimum between phase $0.45<\varphi<0.55$, caused by irradiation.

\section{Line light curves}

In Fig. 8 we show the light curves of the same lines as shown in Fig. 3 at a phase resolution of $0.01 P_{\text {orb. }}$. All lines show a similar behaviour, with the possible exception of He II $\lambda 4686$. The light curve of this line, however, is very similar to that of the continuum. The decrease of light in the Balmer lines during eclipse is very moderate and much less pronounced than that of the continuum, which already indicates that most of the line emitting region remains visible during eclipse.

To explain in more detail the behaviour of the lines, we show an expanded view of the light curve of $\mathrm{H} \beta$, at 0.005 phase intervals, and a schematic view of the light curve in Fig. 9.

Between phases $0.3<\varphi<0.6$ the light curve is roughly constant. At phase $\varphi \sim 0.65$ a decrease in the line intensity starts. This decrease is part of a very wide $(0.6<\varphi<0.2)$, slightly skewed, V-shaped feature, that is centered on $\varphi \sim 0.95$. This phase interval and its center coincides with the visibility of a radiating hot-spot on the rim of a geometrically thick accretion disk (e.g. as in IP Peg, Marsh 1988; Groot 1999). Marsh (1988) and Groot et al. (2001) have shown the hot-spot to be typically around $10000 \mathrm{~K}$, corresponding to a late B, early A-type spectrum. This spectral type is characterized by very strong absorption lines of the Balmer series. We therefore expect the hot-spot region in RW Tri to be the source of strong Balmer (and He I) absorption lines. We conclude that the broad V-shaped feature between $0.6<\varphi<0.2$ is due to the visibility of these strong absorption lines in the hot spot radiation.

Between phase $0.95<\varphi<0.05$ the line intensity increases again, and in the higher Balmer series ( $\mathrm{H} \delta$ and up) actually reaches emission levels that are higher than outside eclipse. 


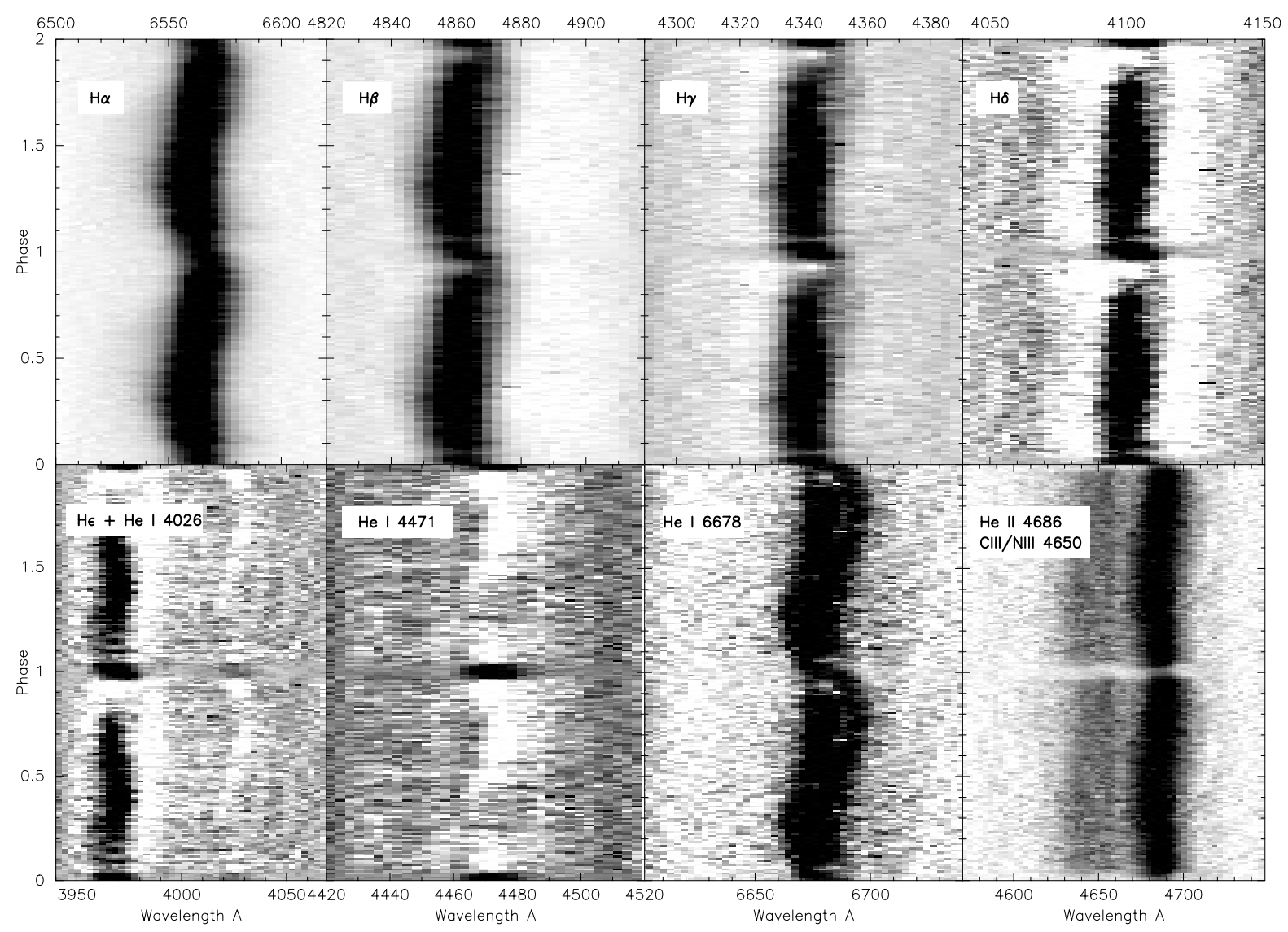

Fig. 3. The trailed spectra (in bins 0.01 in phase) for $\mathrm{H} \alpha, \mathrm{H} \beta$ (plus $\mathrm{He} \mathrm{I} \lambda 4921, \mathrm{H} \gamma$ and $\mathrm{H} \delta$ on the top row and $\mathrm{H} \epsilon$ (plus $\mathrm{He} \mathrm{I} \lambda 4026$ ), $\mathrm{He} \mathrm{I} \lambda 4471$, He I $\lambda 6678$, and He II $\lambda 4686$ plus CIII/NIII on the bottom row. Black indicates emission.

This phase-interval coincides with the eclipse of the accretion disk, and we conclude that the rise in line intensity is due to the fact that the hot spot and accretion disk continuum, with its deep absorption lines, is eclipsed during this phase interval. That the intensities in the higher Balmer lines reach higher levels than outside eclipse is because their line strength is a combination of isotropic emission from an extended, optically thin, region, anisotropic absorption from the hot spot and isotropic absorption from the rest of the disk. During eclipse both absorption components are occulted, leaving solely the emission.

At phase interval $0.98<\varphi<0.02$ the line intensity decreases slightly again. This is seen for $\mathrm{H} \beta$ in Fig. 9, but the same behaviour is also shown by the other lines (as seen at lower phase resolution in Fig. 8). This short-lasting decrease can only be due to the eclipse of the emission site itself. The brief central dip is off-centered with the respect to the line connecting the secondary star with the white dwarf, very similar in phasing to the hot spot. We therefore conclude that the main emission region of both the Balmer lines as well as the HeII 4686 line and the Bowen blend is centered on the hot spot region, which was also concluded from the offset in the radial velocity curves.

\section{Spectral eclipse mapping}

For the eclipse mapping procedure, we used the run-combined light curve to obtain sufficient phase resolution and phase coverage. Analysis of the light curves showed that the profile of

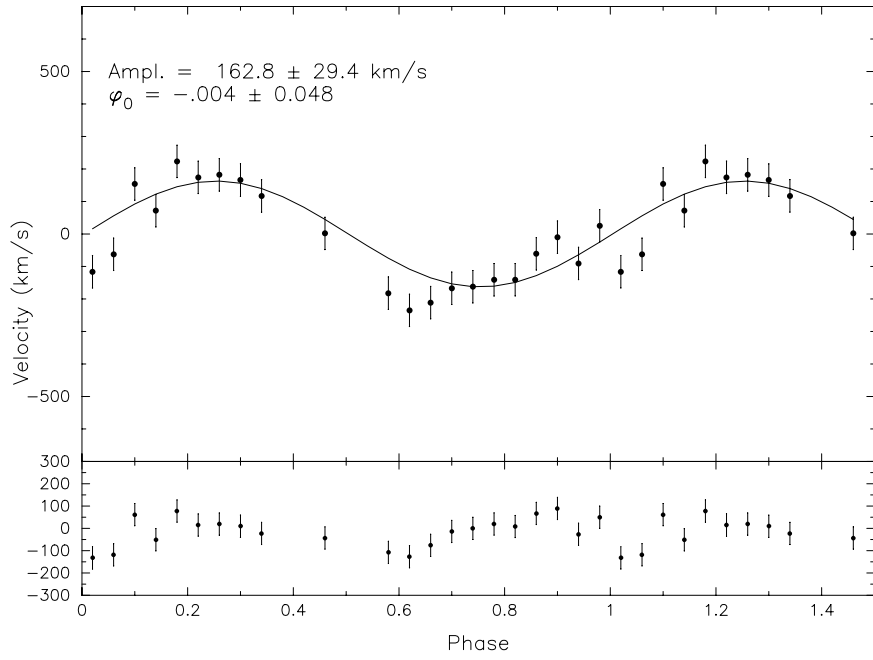

Fig. 4. The radial velocity curve of the Ca I absorption line at $6162 \AA$. The phasing and amplitude of the radial velocity curve correspond well with a place of origin on the secondary star. The decrease in the absorption strength as seen in Fig. 7 prevents a determination of the radial velocity around phase 0.5 .

the eclipse of October 24 deviated in its shape from the rest of the eclipse profiles, especially in the steepness of the egress feature. This eclipse profile has therefore been excluded from the run-combined average. 

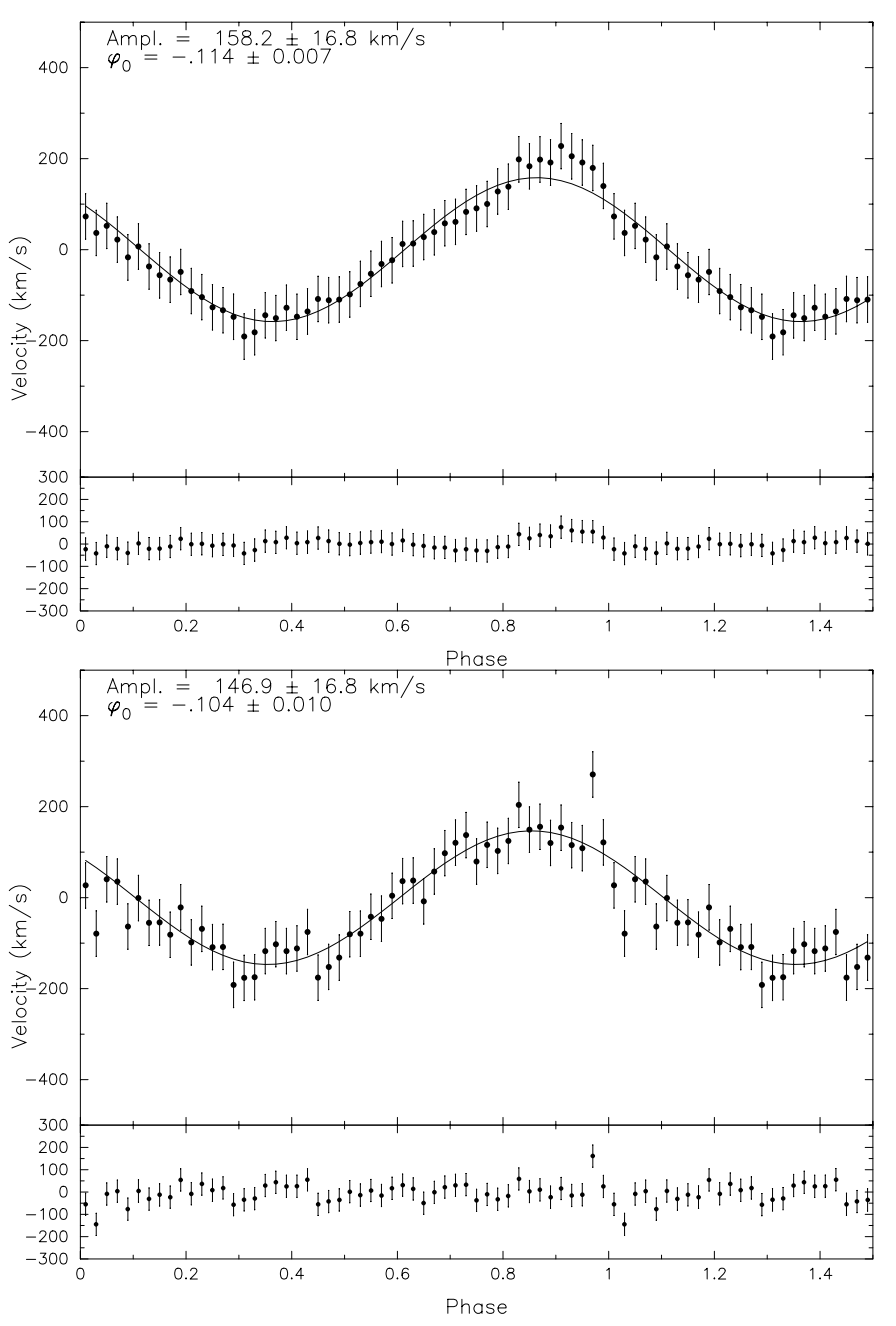

Fig. 5. The radial velocity curve of $\mathrm{H} \alpha$ (top) and He II $\lambda 4686$ (bottom) and a sinusoidal fit to the phase interval $0.1<\varphi<0.9$.

We have straightened the out-of-eclipse profiles by fitting a spline curve to the phases $\varphi<-0.12$ and $\varphi>0.12$, and also brought the light curves to a common scale, which was chosen to be the brightness of RW Tri on the first night at $A B=13.0$ at $4500 \AA$.

The phase-resolved spectra of RW Tri have been divided in 80 narrow band light curves, each $40 \AA$ wide, except around the spectral lines, which were taken as single bins. In Fig. 10 we show the corrected light curves in three narrow band wavelength regions, spanning the wavelength range covered. We see that, despite the variation in the level of the out-of-eclipse light, the eclipse profiles themselves are very similar. The amount of scatter on the narrow-band light curves increases when going to the red. This is most likely caused by an inaccurate slit correction in the red part of the spectrum.

For the eclipse mapping reconstruction we have used a $51 \times$ 51 pixel map, phasebins of 0.005 in phase and the system parameters as given in Table 1.

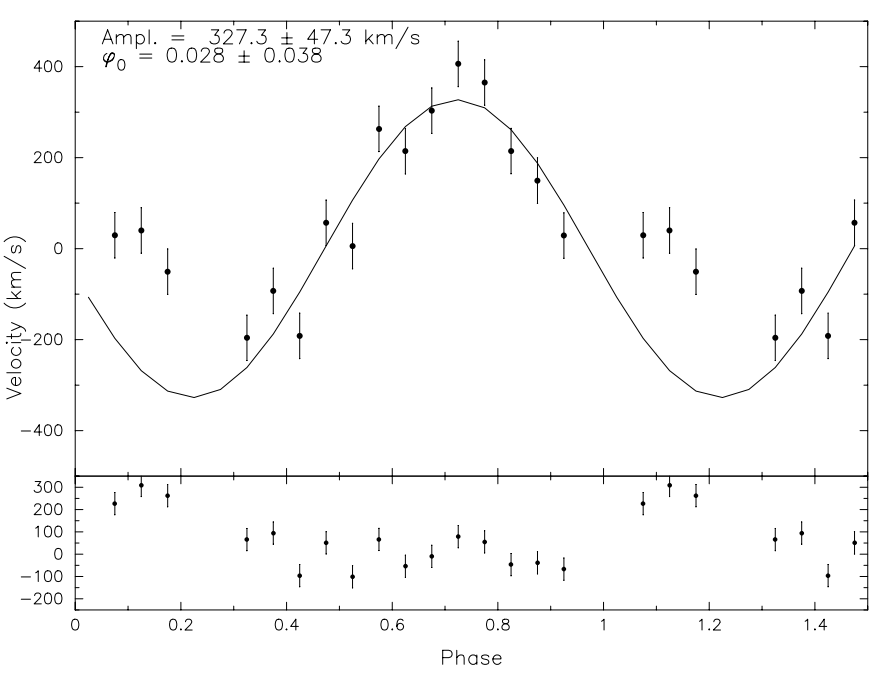

Fig. 6. The radial velocity curve of He I $\lambda 4471$, determined by autocorrelation with the profile at $0.65<\varphi<0.7$. The sinusoidal fit has been made to the phase interval $0.35<\varphi<0.9$.

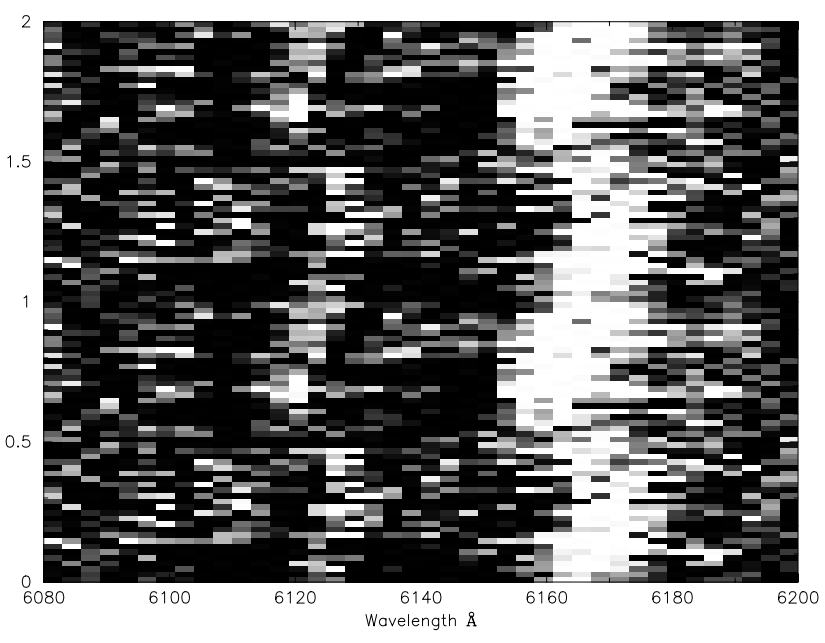

Fig. 7. Trailed spectrogram of the Ca I triplet between 6100-6200 A. The Ca I $\lambda 6162$ is the strongest of the three, and the others are located at $\lambda 6121$ and $\lambda 6102$. This last one is only slightly visible.

\subsection{Disk size}

To measure the size of the accretion disk at different wavelengths we have used the distance $\left(R_{0.1}\right)$ where the intensity on the disk has fallen to $10 \%$ of the central intensity. This measure was used by Rutten et al. (1992) to compare the relative sizes of the disks in six different novalike systems. We find from our eclipse maps that $R_{0.1}=0.25 \pm 0.10 R_{L_{1}}$ at $4420 \AA$. The rather large error is caused by a relatively flat run of the reconstructed intensity with radial distance at this wavelength. For $6270 \AA$ the disk size has increased to $R_{0.1}=0.45 \pm 0.05 R_{L_{1}}$. Both values are comparable to the values found by Rutten et al. (1992) at $4410 \AA\left(R_{0.1}=0.28 \pm 0.03 R_{L_{1}}\right)$ and $8010 \AA\left(R_{0.1}=0.43 \pm\right.$ $\left.0.03 R_{L_{1}}\right)$. 


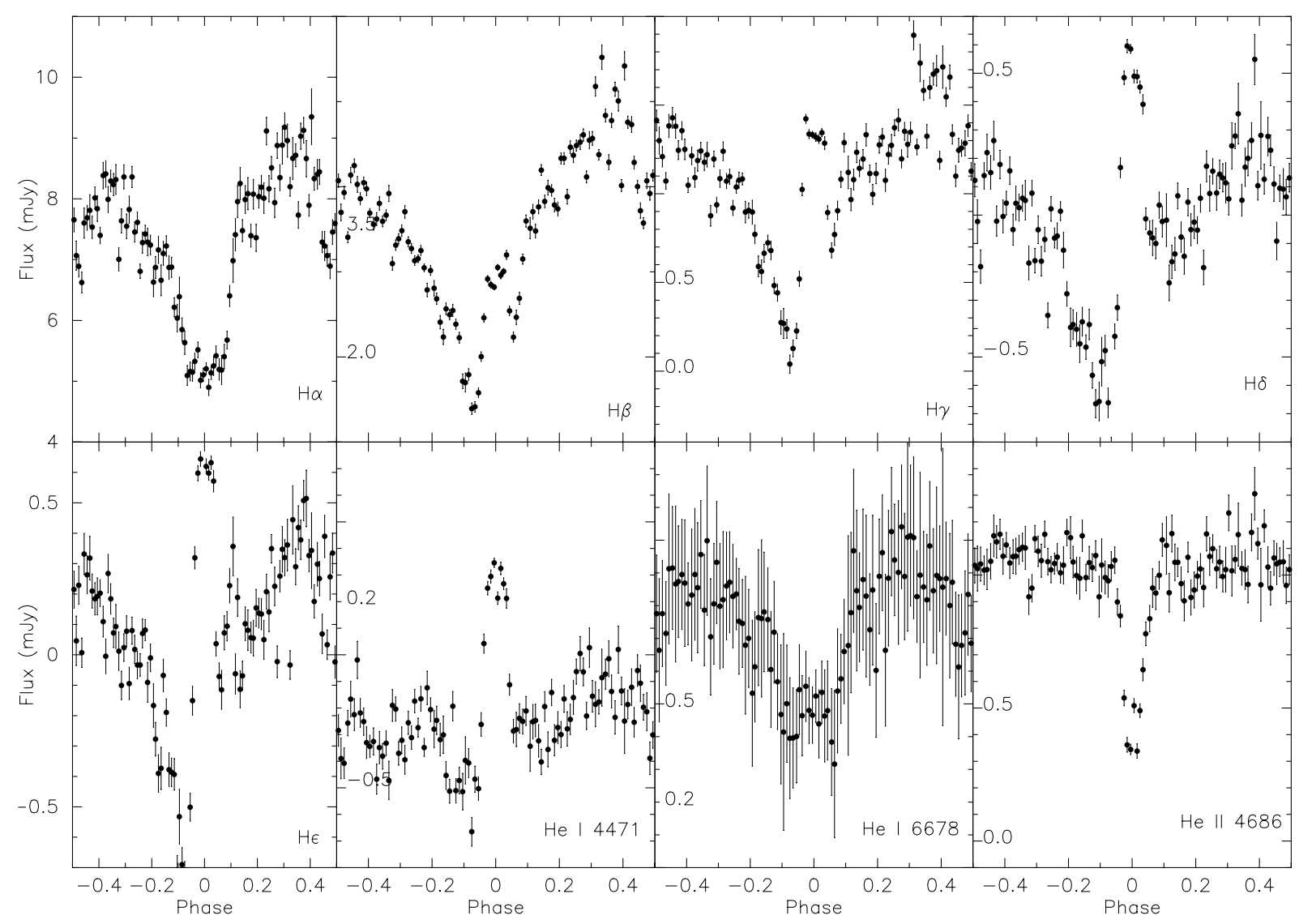

Fig. 8. The light curves of the same lines as showed in Fig. 3.

\subsection{Accretion disk annuli spectra}

We have defined seven regions in the accretion disk of RW Tri, labeled "A" through "G" at radii of $0.1,0.2,0.3,0.4,0.5$, $0.7 R_{L_{1}}$ and a hot-spot region ranging from $0.2-0.7 R_{L_{1}}$ and $0.8<\varphi<0.03$ (Fig. 11). The spectra of these regions are shown in Fig. 12. We see that there is a strong change in the slope of the spectrum from blue near the white dwarf to red in the outer parts of the accretion disk. No clear signature of any spectral lines (apart perhaps from the Balmer series in absorption in region "A") is seen. Not surprisingly the non-eclipsed light (region " $\mathrm{H}$ ") shows the spectral lines $(\mathrm{H} \alpha$, HeI $\lambda 5875$, $\mathrm{H} \beta$, He II $\lambda 4686, \mathrm{H} \gamma$ and $\mathrm{H} \delta$ ) in emission on top of a continuum level that rises to the red.

\subsection{Distance to RW Tri}

In order to convert the reconstructed fluxes to specific intensities, from which temperatures can be derived by e.g. blackbody fitting, it is imperative to have a good estimate of the distance to the system. The distance of RW Tri was recently determined by parallax measurements using the HST Fine Guidance Sensor to be $341 \pm 35$ pc (McArthur et al. 1999).

The distance of RW Tri can also be estimated by allowing the distance as well as the temperature to vary in blackbody fits to the reconstructed accretion disk spectra. Blackbody fitting in the wavelength region 4000-6200 ̊ gives a distance to RW Tri of $330 \pm 40 \mathrm{pc}$, in excellent agreement with the parallax measurements and also with the estimate of the fractional contribution of the secondary by Rutten et al. (1992; $330 \mathrm{pc}$ ). We refer to McArthur et al. (1999) for a comparison with other distance estimates. We will use the value of $330 \mathrm{pc}$ in the further analysis.

\subsection{The radial temperature profile}

To determine the radial temperature profile of the accretion disk we have used the wavelength region of $4000 \AA-6200 \AA$, from which the emission lines have been eliminated. The blue cutoff has been chosen to avoid any influence of the Balmer jump and the red cut-off has been chosen because trial blackbody fits showed that the reconstructed intensities at these wavelengths strongly deviated from the expected values based on the trend in the bluer part of the wavelength which generally agreed well with the blackbody fits.

After the determination of the distance as described in the previous paragraph $(330 \pm 40 \mathrm{pc})$, we fixed the distance at this value and fitted only for the temperature. For this we have taken the spectrum at each pixel (in specific intensities) and fitted a temperature to this spectrum. We show the radial temperature profile of the accretion disk in RW Tri in Fig. 13, which also shows the theoretical predictions for the radial temperature profile based on optically thick, steady state accretion disks. To calculate these theoretical profiles we have used the white dwarf mass as given in Table 1 and the white dwarf mass-radius relation of Nauenberg (1972). 


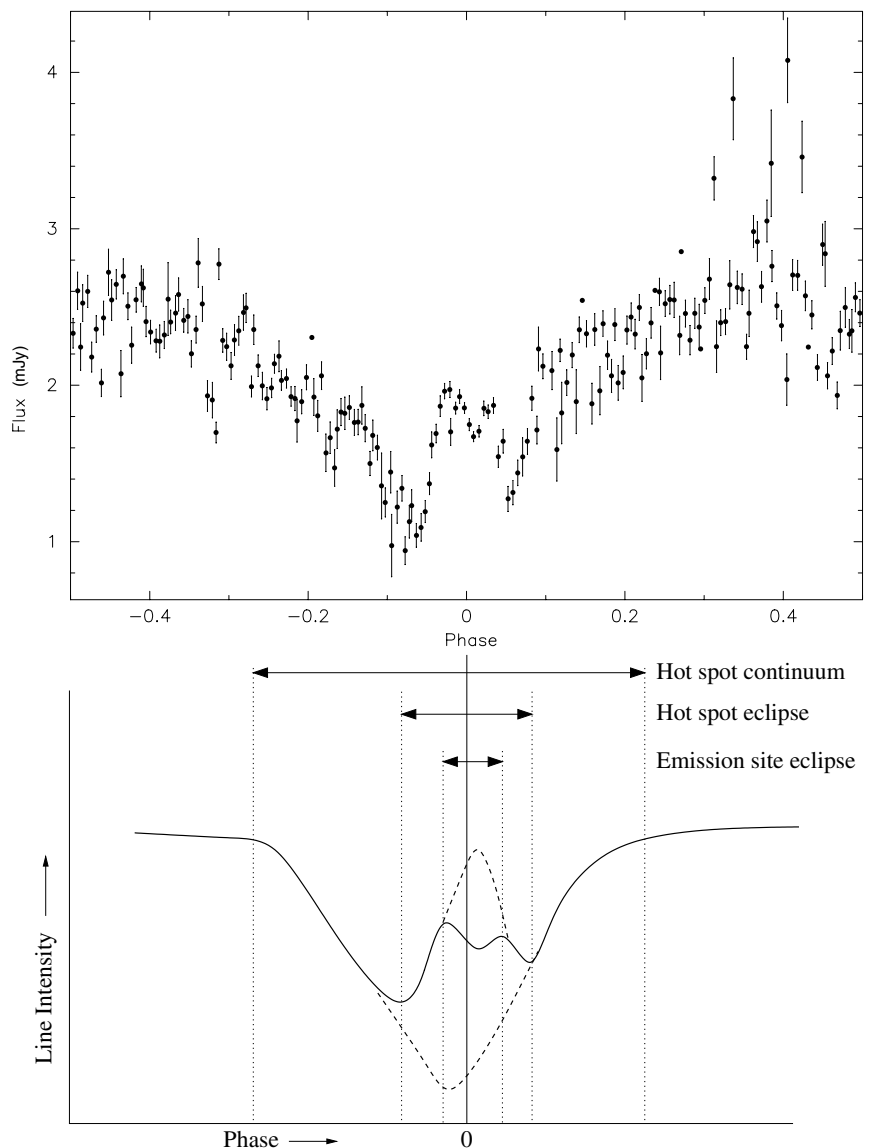

Fig. 9. The light curve of $\mathrm{H} \beta$ at a phase resolution of $0.005 P_{\text {orb }}$ (top), and a schematic view of the light curve, indicating the different components (bottom).

To determine the position of the hot-spot we have taken the ratio of the continuum intensity maps at $4060 \AA$ and one at $6270 \AA$ to maximize the constrast between the and blue hotspot and the cooler outer disk. This ratio shows the position of the hot-spot to be centered on $(r, \varphi)$ of $\left(0.5 R_{L_{1}}, 0.875\right)$.

\section{Discussion}

\subsection{Continuum radiation from the eclipse maps: Mass-transfer rate}

From the wealth of information RW Tri displays in our dataset we construct a conceptual model of the structure of RW Tri (see Fig. 14).

The accretion disk, although showing substantial variation on its radial temperature profile, on average follows the standard, steady state, $T \propto R^{-3 / 4}$, profile predicted from theory and observed in RW Tri before (Rutten et al. 1992), up until a radius of $0.15 R_{L_{1}}$ from the white dwarf, where the temperature profile flattens off. Comparing our temperature profile with those derived by Rutten et al. (1992) on the basis of four-colour photometry and by Horne \& Stiening (1985) on the basis of brightness temperature estimates of their $B$ band photometry, we see that our profile already levels off at a larger distance from the white dwarf (at $\sim 0.15 R_{L_{1}}$ here and at $\sim 0.06 R_{L_{1}}$ in Rutten et al. 1992 and Horne \& Stiening 1985).
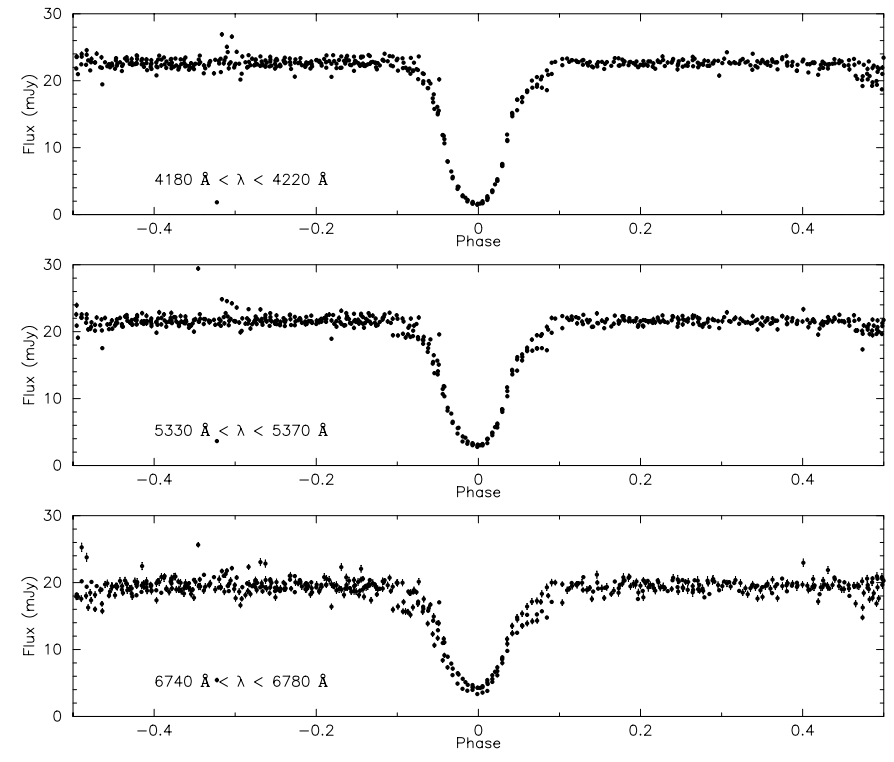

Fig. 10. The run-combined straightened light curves of RW Tri in three wavelength regions centered on $4200 \AA$, $5350 \AA$ and $6760 \AA$.

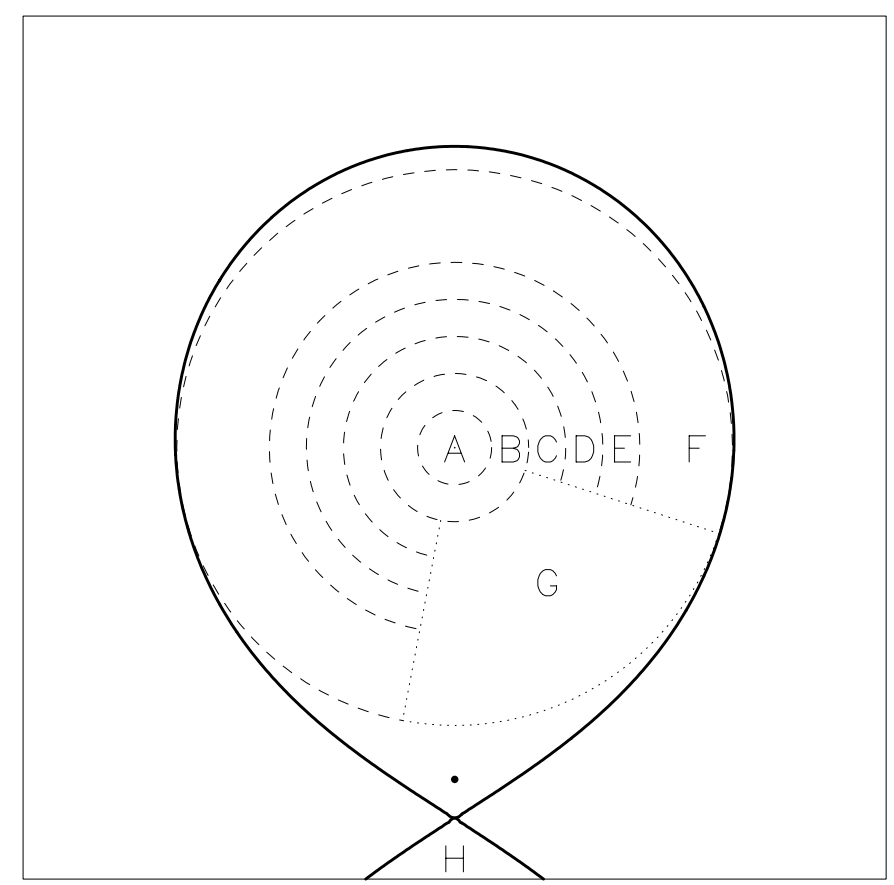

Fig. 11. Schematic view of the white dwarf Roche lobe, showing the subdivision of the Roche lobe in seven regions, labeled "A"-“G". The uneclipsed light is denoted by region " $\mathrm{H}$ ", tentatively placed on the secondary.

From the radial temperature profile between $0.2-0.56 R_{L_{1}}$ we deduce a mass-transfer rate through the disk of $\sim 10^{-8} M_{\odot} \mathrm{yr}^{-1}$. This mass transfer rate is somewhat higher than in Rutten et al. (1992), but this is partly caused by the lower distance $(270 \mathrm{pc})$ used in that study.

\subsection{Line profiles: Velocities and emission sites}

The strong presence of emission lines in the uneclipsed light indicates that they are formed out of the plane of the disk. 


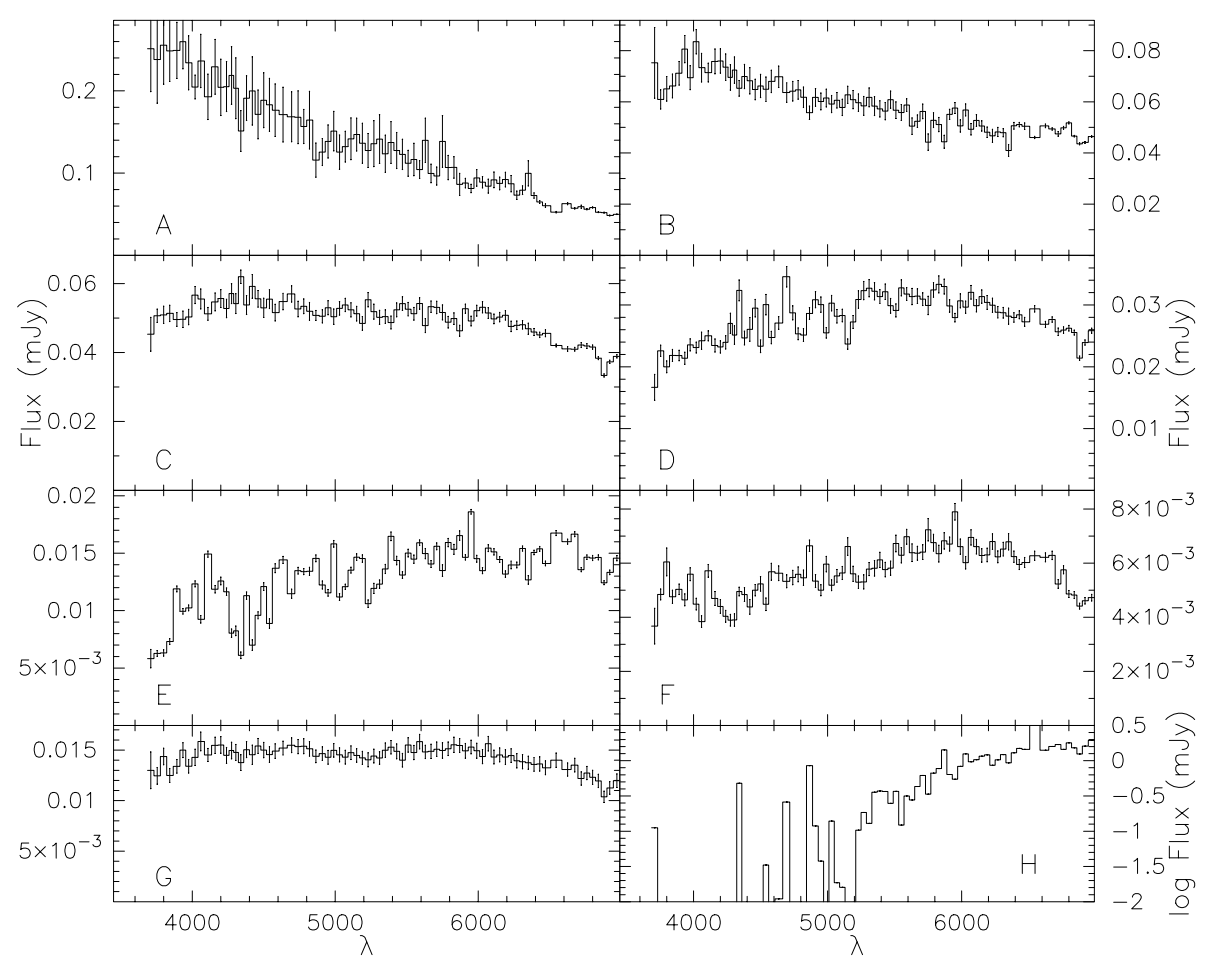

Fig. 12. The reconstructed spectrum of the accretion disk in RW Tri in the regions defined in Fig. 11. Fluxes are given per surface tile element (each $1.6 \times 10^{18} \mathrm{~cm}^{2}$ including the foreshortening at $i=75^{\circ}$ ). We see that the slope of the spectrum changes dramatically from very blue in the inner parts, to red in the outer parts. The hot-spot area (region "G") is more blue than the rest of the outer disk. The fluxes of the uneclipsed light component (region "H") are plotted on a logarithmic scale, all others on a linear scale.

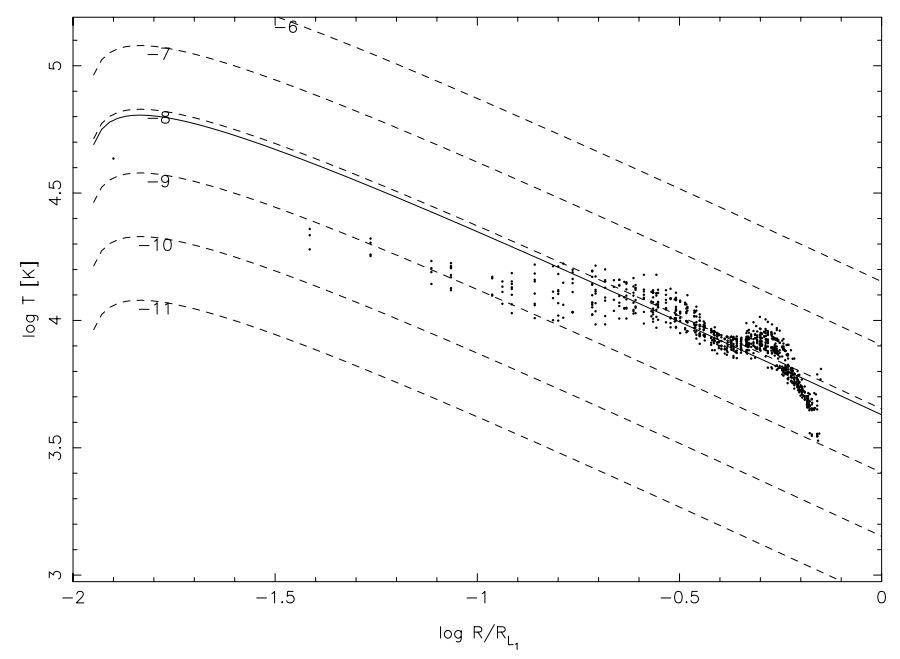

Fig. 13. The radial temperature profile of RW Tri, as deduced from the spectral eclipse mapping. The dashed lines show the theoretical prediction of the radial temperature profile based on the theory of optically thick, steady state accretion disks.

The fact that during eclipse an emission component appears in He I $\lambda 4026$ and $\lambda 4471$, suggests that the absorption we see during most of the orbit is a combination of absorption and emission, where the absorption is caused by material close to the disk, which is back-lit by the white dwarf and surroundings, and the emission is caused by material higher above the disk, which is, in our line-of-sight, not back-lit by material behind it. This would also explain why the He I $\lambda 6678$ line is in

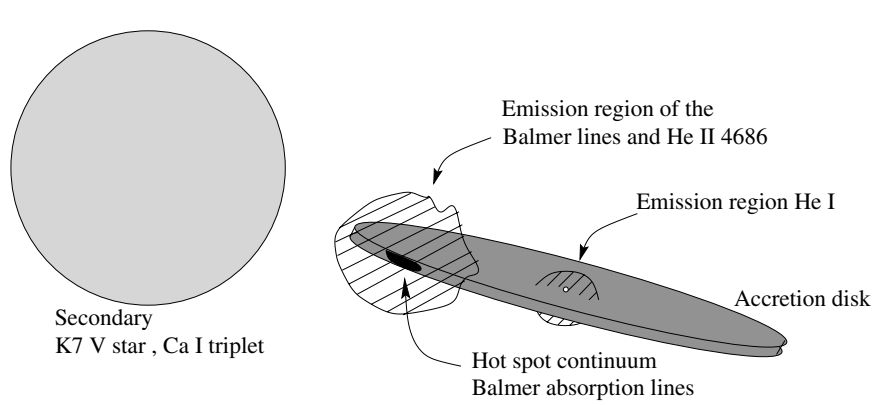

Fig. 14. Schematic view of the structure of RW Tri and the location of the areas producing the spectral lines discussed in the text.

emission, since this is the line with the lowest excitation level and therefore presumably the largest emission region.

In the trailed spectra the emission lines are characterized by a high and low velocity component. The phasing of the highvelocity component indicates that the component originates on the line of centers and on the white dwarf side of the centerof-mass. The amplitude is too low to be connected with any rotational velocity in the accretion disk (which is assumed to have a Keplerian rotation field, with a minimum velocity of $\sim 500 \mathrm{~km} \mathrm{~s}^{-1}$ in the outer regions of the disk). Considering both these two facts (no phase lag and an amplitude that is too low for rotation of the accretion disk) it appears that the white dwarf itself or its immediate surroundings is the place of origin of the high velocity component. In this case the (uncertain) amplitude of $330 \mathrm{~km} \mathrm{~s}^{-1}$ would reflect the orbital velocity of the white dwarf. 
The radial velocity curve of the low-velocity component shows a significant offset with respect to the line of centers, and this phase lag puts it at the position where the hot-spot is located. The amplitude also coincides with the velocity of the hot-spot region in the binary frame. This means that both the gas at the emission site of the low-velocity component, as well as that at the emission site of the high-velocity component must be decoupled from the Keplerian flow in the disk.

Overall it appears that the spectral lines are dominated by two emission regions that both have appreciable vertical extension with respect to the disk. The most dominant of these region is located at the hot spot (located at $(r, \varphi)=0.5 R_{L_{1}}$, 0.875 ), as evidenced by the phase lag, radial velocity amplitude and light curve of the Balmer and $\mathrm{He} \mathrm{I}$ emission lines. Also the He II $\lambda 4686$ line (and the CIII/NIII Bowen blend) is formed in this region, as evidenced by the identical radial velocity curves of $\mathrm{H} \alpha$ and $\mathrm{He}$ II $\lambda 4686$. The marked difference in the $\mathrm{H} \alpha$ and He II $\lambda 4686$ line light curves, where the $\mathrm{H} \alpha$ light curve shows a very broad absorption lasting from $0.6<\varphi<0.2$, whereas the He II $\lambda 4686$ light curve is very similar to that of the continuum is caused by the fact that the hot spot is too cool to cause appreciable anisotropic He II $\lambda 4686$ absorption, and the He II $\lambda 4686$ light curve therefore shows "just" the eclipse by the secondary star. Also, the He II $\lambda 4686$ emission region is much smaller than the Balmer region, since no central emission is seen at mid-eclipse.

\subsection{SW Sex behaviour}

During our observations RW Tri showed some features that are commonly used as identifiers of the SW Sex sub-class of novalike CVs (see Thorstensen et al. 1991 and Groot et al. 2001): single-peaked emission lines, low amplitude radial velocity curves of the main Balmer lines, phase lags of the Balmer lines, shallow eclipses of the Balmer lines, a flat radial temperature profile and He II $\lambda 4686$ emission. Although some of these indicators may be more hinted at in our observations than firmly proven, the combined occurence of these indicators raises the question whether RW Tri should be considered to be an SW Sex star.

Groot et al. (2001) showed that in recent observations of SW Sex in a low state, this system lacked some of the SW Sex features, e.g. the phase 0.5 absorption. Combined with the RW Tri observations presented here it appears that the boundary between UX UMa-, RW Tri- and SW Sex-like novalikes (see Warner 1995 for definitions) is vague and depends on the brightness of the system at the moment of observation. It appears that the behaviour that is "standard" for the sub-classes are the extremes of a sliding scale, most likely depending on the mass-transfer rate, where the SW Sex behaviour becomes more prominent with increasing mass-transfer rate.

Acknowledgements. P.J.G. whishes to thank Alex de Koter for pleasant discussions on the line formation mechanisms in non-LTE regimes, and Claudio Moreno and the staff of the ING observatory for their hospitality during various visits. We would also like to thank the referee, dr. Bruch, for his very useful comments and Janet Drew for reading and commenting on the manuscript. P.J.G. acknowledges partial support by NWO Spinoza grant 08-0 to E. P. J. van den Heuvel and an NWO-VIDI grant 639.042.201 to P. J. Groot. The Isaac Newton Telescope is operated by the Isaac Newton Group in the Spanish Observatorio del Roque de los Muchachos of the Instituto de Astrofísica de Canarias.

\section{References}

Cardelli, J. A., Clayton, G. C., \& Mathis, J. S. 1990, ApJ, 345, 245

Frank, J., King, A. R., \& Raine, D. 1992, in Accretion Power in Astrophysics, CAS 21 (Cambridge, UK: CUP)

Groot, P. J., Augusteijn, T., Barziv, O., \& van Paradijs, J. 1998, A\&A, 340, L31

Groot, P. J. 1999, Ph.D. Thesis, University of Amsterdam

Groot, P. J., Rutten, R. G. M., \& van Paradijs, J. 2001, A\&A, 368, 183

Horne, K., \& Stiening, R. F. 1985, MNRAS, 216, 933

Horne, K. 1986, PASP, 98, 609

Kaitchuck, R. H., Honeycutt, R. K., \& Schlegel, E. M. 1983, ApJ, 267, 239

Marsh, T. 1988, MNRAS, 213, 1117

McArthur, B. E., Benedict, G. F., Lee, J., et al. 1999, ApJ, 520, L59

Mason, K. O., Drew, J. E., \& Knigge, C. 1997, MNRAS, 290, L23

Nauenberg, M. 1972, ApJ, 175, 417

Oke, J. B. 1990, AJ, 99, 1621

Protitch, M. 1937, Bull. Astr. Obs. Belgrade, 9-10, 38

Robinson, E. L., Shetrone, M. D., \& Africano, J. L. 1991, AJ, 102, 1176

Rutten, R. G. M., van Paradijs, J., \& Tinbergen, J. 1992, A\&A, 260, 213

Rutten, R. G. M., Dhillon, V. S., Horne, K., Kuulkers, E., \& van Paradijs, J. 1993, Nature, 362, 518

Rutten, R. G. M., Dhillon, V. S., Horne, K., \& Kuulkers, E. 1994, A\&A, 283, 441

Smak, J. 1995, ActA, 45, 259

Smith, R. C., Cameron, A. C., \& Tucknott, D. S. 1993, in Cataclysmic Variables and Related Physics, ed. O. Regev, \& G. Shaviv (Bristol: IoP), 70

Still, M. D., Dhillon, V. S., \& Jones, D. H. P. 1995, MNRAS, 273, 849

Thorstensen, J. R., Ringwald, F. A., Wade, R. A., Schmidt, G. D., \& Norsworthy, J. E. 1991, AJ, 102, 272

Walker, M. F. 1963, ApJ, 137, 485

Warner, B. 1995, Cataclysmic Variable Stars, CAS 28 (Cambridge, UK: CUP) 